\title{
SILVIO PALAZZI (1892-1979), UN PIONIERE DELLA MODERNA ODONTOIATRIA ITALIANA
}

\author{
SILVIO PALAZZI (1892-1979), \\ A PIONEER OF MODERN ITALIAN DENTISTRY
}

\author{
Paolo Zampetti", Andrea Scribante**
}

\section{RIASSUNTO}

Nonè facile analizzare una figura complessa come quella di Silvio Palazzi (I892-I979) (fig.I). Senza dubbio fu uno dei personaggi di maggior spicco nel panorama odontostomatologico italiano per circa un cinquantennio, uno dei protagonisti della trasformazione dell'Odontoiatria pionieristica a in quella scientifica, un precursore ed un uomo con una mentalità aperta, dotato di una visione lungimirante. Personalità eclettica, versatile, da certi punti di vista addirittura geniale ma anche imprevedibile, fu al centro della vita accademica e professionale dell'Odontoiatria italiana; pochi possono vantare un'attività didattica, clinica, scientifica come la sua. Divenuto, in età giovanissima, direttore di una clinica che era ancora poco piu che un ambulatorio seppe portarla ad un livello di eccellenza che non aveva riscontri in Italia (fig. 2) e che poteva essere paragonato a quello delle grandi cliniche odontoiatriche europee.

Fu autore di un "Trattato di Odontologia" (fig. 3 e 4) che ebbe sette edizioni, sui cui si formarono intere generazioni di dentisti, e di oltre cinquecento pubblicazioni scientifiche, in

* Dipartimento di Scienze Clinico Chirurgiche, Diagnostiche e Pediatriche - Corso di Laurea Magistrale in Odontoiatria e Protesi Dentaria- Insegnamento di Storia dell'Odontoiatria, Università degli Studi di Pavia, Italy. E-mail: paolo.zampetti@unipv.it. ORCID: 0000-0003. 4399-7034.

** Dipartimento di Scienze Clinico Chirurgiche, Diagnostiche e Pediatriche - Corso di Laurea Magistrale in Odontoiatria e Protesi Dentaria- Insegnamento di Patologia Speciale Odontostomatologica, Università degli Studi di Pavia, Italy. E-mail: andrea.scribante@unipv.it. ORCID: 0000-0002-2760-0124.

Correspondence Address: Andrea Scribante, Sezione di Odontoiatria, Dipartimento di Scienze Clinico Chirurgiche, Diagnostiche e Pediatriche, Università degli Studi di Pavia, Piazzale Golgi 2, 27100 Pavia, Italy. E-mail: andrea.scribante@unipv.it. 
tutti i campi dell'Odontostomatologia; predilesse particolarmente le indagini istologiche ed istochimiche, come spesso ricordava, per avere avuto una preparazione impostata in tal senso dalla sua frequenza presso l'istituto di Patologia Generale di Pavia diretto da Camillo Golgi (I843-1926, Premio Nobel per la Medicina nel I906). In campo clinico ogni settore della Odontoiatria lo vide attento ed appassionato cultore, in particolare dell'Endodonzia e della Parodontologia. Inoltre, fu un pioniere dell'Implantologia quando questa branca riscuoteva più critiche che successi ed iniziò le ricerche sull'azione profilattica del fluoro quando molti erano contrari. Si batté assiduamente per una differente legislazione odontoiatrica: fu un convinto sostenitore di un Corso di Laurea apposito per la preparazione del futuro odontoiatra, già sin dagli anni Cinquanta: poiché questo progetto sembrava di difficile realizzazione, propose se non altro l'obbligo di una specializzazione post-laurea per garantire una formazione idonea. Accanto a ciò, per il suo modo di porsi spesso aggressivo e polemico si alienò l'amicizia di molti colleghi e si creò numerosi nemici. Certamente fu un personaggio che non può passare inosservato e che merita, ad oltre quaranta anni di distanza dalla morte, una attenta valutazione storica.

Parole chiavi: Palazzi, storia dell'odontoiatria, Università degli Studi di Pavia

\section{BREVI CENNI BIOGRAFICI}

Silvio Palazzi (Figura I) nacque a Milano il 2r maggio I892.

Conseguita la maturità classica si era iscritto, nel I9ıI, alla facoltà di Medicina e Chirurgia dell'Università di Pavia. Subito appassionatosi di Istologia e di Embriologia Generale frequentò, già dall'anno successivo, in qualità di allievo interno, l'Istituto di Anatomia Umana Normale, diretto da Luigi Sala (I863-I930); iniziò in questa sede la sua preparazione scientifica che caratterizzò sempre il suo metodo sperimentale nei vari filoni di ricerche in campo odontostomatologico.

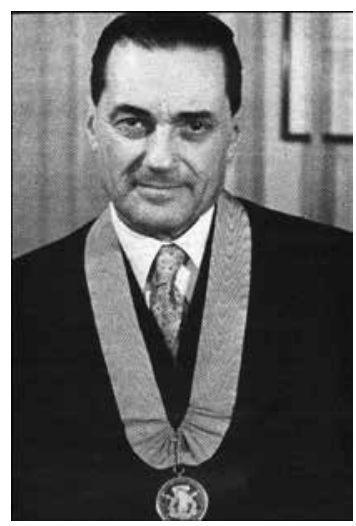

Fig ı. Silvio Palazzi (1892-1979) nel I958. Si nota al collo la Medaglia Teresiana, che connota i professori ordinari dell'Università di Pavia. 
Nel 1917 si laureò presso l'Università di Padova, ove si era trasferito in quanto richiamato alle armi presso il locale distretto, frequentando l'Università Castrense di S. Giorgio al Nogaro. Terminato il primo conflitto mondiale, il Palazzi si iscrisse alla Scuola di Perfezionamento in Odontoiatria dell'Istituto Stomatologico di Milano, dove si diplomò nel 1920 a pieni voti assoluti con lode (Beretta, I935; Branchini, 1933, I940; Palazzi, 1939).

Contemporaneamente frequentava l'Istituto di Odontoiatria dell'Università di Pavia, diretto da Ludovico Coulliaux (I863-1929), che lo ebbe caro come un figlio e che fece di lui l'allievo prediletto (Palazzi, 1939; Zampetti, 200I, 2005).

Nel 1923 ottenne la libera docenza in Odontoiatria e Protesi Dentaria e per i due successivi anni accademici tenne il corso libero di Radiologia Orale presso l'Università di Pavia. Diresse la Clinica Odontoiatrica dell'Università di Pavia dal I925 al I962 (Figura 2).

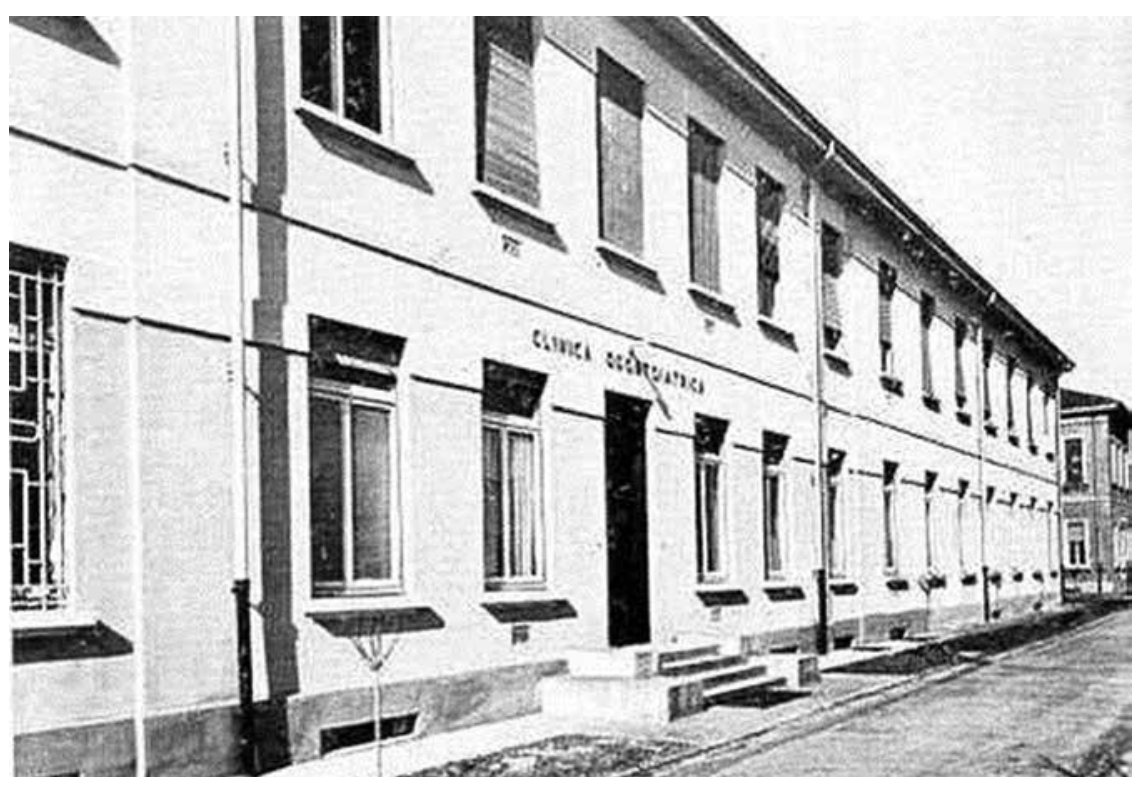

Fig 2. Facciata della clinica odontoiatrica dell'Università di Pavia negli anni '5o.

Nel 1947 venne nominato direttore dell'Istituto Stomatologico Italiano di Milano e Direttore della Scuola di Specializzazione in Odontoiatria ivi annessa. Ottenne il Premio Miller, ambito riconoscimento che premiava i migliori odontoiatri mondiali, nel I952 (Palazzi, 1939; Zampetti, 200I).

Morì a Como il ız ottobre 1979. 


\section{L'OPERA IN SINTESI}

La parabola ascendente del Palazzi incomincerà nel 1925, quando, in seguito alle dimissioni del suo maestro Ludovico Coulliaux venne chiamato, appena trentatreenne, a succedergli, ottenendo contestualmente la direzione dell'Istituto di Odontoiatria (Zampetti, 200I, 2005).

E'opportuno in questa sede ricordare brevemente la figura del Coulliaux: fu il secondo Libero Docente di Clinica Odontoiatrica in Italia (presso l'Università di Parma) nel I893.

Nel I9o9 ottenne l'incarico di insegnamento della medesima materia presso l'Università di Pavia, dove venne immediatamente apprezzato per l'umanità e per le sue notevoli capacità didattiche.

Erano anni, quelli, in cui l'Odontoiatria italiana faticava ad affermarsi: ma Coulliaux, con tenacia e perseveranza, contribuì, assieme a poche altre elette personalità (fra le quali spicca quella di Carlo Platschick (I853-I9I2) primo Libero Docente in Italia di Odontoiatria) all'elevazione morale, medica e scientifica di tale disciplina. Nel Igı2 riuscì a fondare, fra enormi difficoltà, l'Istituto di Odontoiatria dell'Università di Pavia, prima clinica universitaria in Italia, di cui fu primo Direttore, carica che mantenne per dodici anni, dimettendosi anzitempo per motivi di salute e facendo in modo di lasciare la sua eredità appunto al suo allievo prediletto, Silvio Palazzi.

Ottenuta la direzione, quest'ultimo provvide a gettare le basi per una riorganizzazione generale dell'Istituto da un punto di vista clinico, scientifico, didattico e sperimentale.

Il contributo che la clinica pavese iniziò ad apportare in termini di ricerca e di clinica in quegli anni fu di fondamentale importanza per lo sviluppo futuro dell'Odontoiatria italiana.

Ricorderemo di questo primo periodo in particolar modo gli studi iniziati dal Palazzi e dalla sua scuola sulle paradenziopatie, sulla terapia radicolare, sull'impiego di gas anestetici, sulle tecniche chirurgiche di avulsione. Furono avviate anche indagini in campo istologico ed istopatologico dei tessuti orali.

Già a partire dal 1925 il Palazzi aderì alla più importante associazione tedesca per lo studio della malattia parodontale, la Arbeitsgemeinschaft fur Paradentoseforschhung, che si basava sugli studi effettuati da Gottlieb, Karoly, Orban e Weschi (Palazzi, r962; Zampetti, 2003).

Uno dei filoni di ricerca impostati dal Direttore e dai collaboratori fu dunque relativo alla rielaborazione del concetto di "piorrea alveolare", che portò 
alla creazione, nel 1927, da parte dello stesso Palazzi, del termine "paradenziopatie"; istituì nuova nomenclatura, nuova sistematica clinica, nuove indagini istopatologiche e moderni concetti terapeutici (Palazzi, r962).

Il Palazzi fu membro dell'Associazione Internazionale per gli studi sul Paradenzio (ARPA) e fu fondatore della sezione italiana (ARPA italica) di cui fu per anni presidente ed organizzatore di vari convegni; l'attività di ricerca in tale settore fu forse quella che maggiormente appassionò il Palazzi e la sua scuola per tutto il periodo della sua direzione (Palazzi, I962; Zampetti, 2003). $\mathrm{Ne}$ fu espressione la partecipazione a numerosi congressi tenutisi in Italia ed all'estero per il trentennio successivo.

Altro campo nel quale profuse le sue energie fu quello della odontoiatria conservativa, e dell'endodonzia. In questo campo i suoi contributi furono molteplici: innanzitutto propose il concetto di sterilizzazione di tutto lo strumentario endodontico e la realizzazione del trattamento endodontico in asepsi.

E' da ricordare che intorno al 1925 , specie in Italia, le indagini radiologiche erano ancora poco diffuse, cosicché coloro che esercitavano la terapia endodontica non avevano possibilità di controllo sulla entità del processo patologico, né sui risultati terapeutici; il Palazzi propose una linea di condotta basata sul controllo radiografico pre e post-intervento. L'impiego sistematico delle radiografie nella diagnosi e nel controllo dei denti trattati, già affetti da gravi lesioni periapicali, permise di valutare la possibilità di restituito ad integrum dopo una corretta terapia endodontica. Palazzi introdusse, a tal proposito, in Italia, la colloid-terapia, vale a dire il trattamento e la medicazione dei canali con una sostanza composta di argento colloidale e iodoformio.

Ideò inoltre, nella branca chirurgica, originali metodiche per l'avulsione dentale e propose, per primo in Italia, una particolare tecnica per la riduzione delle fratture maxillo-facciali (Zampetti, 2003, 2006; Zampetti e Mapelli, 2004). Negli anni Trenta il Palazzi ottenne, dal Ministero dell'Educazione Nazionale, di poter attivare la Scuola di Specializzazione in Odontoiatria e Protesi Dentaria (la seconda in Italia) presso la Clinica da lui diretta; in questa sede, con l'aiuto dei liberi docenti dell'Istituto, vennero impartite lezioni a carattere teorico-pratico nelle varie branche della stomatologia, anche mediante l'ausilio di pellicole in bianco-nero e a colori in tempi in cui questa metodologia era ancora poco usata.

Gli specializzandi potevano poi esercitarsi in appositi laboratori dotati di manichini simulanti il paziente; anche in ciò l'Istituto di Pavia fu all'avan- 
guardia essendo fra i primi in Italia a proporre una equilibrata fusione fra teoria e propedeutica (Zampetti, 2006, 20IIa, 20Irb).

L'opera di Palazzi, coadiuvato dal suo Aiuto Cinzio Branchini (1903-1993), fu orientata verso una attività didattica sicuramente all'avanguardia: essa era basata su di una impostazione teorico-pratica della Scuola e della Clinica, che prevedeva l'istituzione di vari corsi, tenuti da liberi docenti dell'Istituto (Amodeo, Babini, Bracchetti, Bugliari, Caudana, Hruska, Zerosi) su vari argomenti dell'odontostomatologia, fra cui conservativa, protesi, chirurgia, anestesia e istologia. Da un punto di vista clinico, nel 1937 fu il primo in Italia a proporre una terapia ortodontica con metodo biomeccanico.

Incombeva però il secondo conflitto mondiale; il Palazzi, già arruolatosi volontario per la campagna di Etiopia nel 1936, venne chiamato successivamente al fronte in Africa Orientale; la direzione provvisoria dell'Istituto fu assunta dall'Aiuto Cinzio Branchini che del Palazzi era, come già detto, il più stretto collaboratore. Una interessante testimonianza di tale periodo è contenuta in un archivio fotografico, che documenta l'attività del Palazzi in Eritrea; il fondo si compone di circa 230 fototipi (200 positivi e 30 immagini digitali) e venne donato da Silvio Manicardi, nipote del Nostro, alla Società Geografica Italiana (sito internet in nota).

Negli anni Quaranta fu l'iniziatore nella Clinica Pavese di studi sulle resine sintetiche per corone e ponti; contemporaneamente dette particolare impulso allo studio sperimentale della istologia e dell'istopatologia del dente e della sua innervazione. In collaborazione con la Clinica Medica diretta da Adolfo Ferrata sì indagò nel campo delle emopatie, delle malattie degli organi e sistemi della vita vegetativa e di relazione e relative influenze sulla polpa dentale, dimostrando che i soggetti affetti da gravi malattie generali presentano anche alterazioni della polpa (Zampetti, 2003, 2006, 20II; Zampetti e Mapelli, 2004).

Per tutti gli anni Cinquanta i filoni di ricerca iniziati dal Palazzi furono variamente orientati: ricordiamo particolarmente le ricerche sulla prevenzione della carie dentale mediante fluoro contenuto in paste dentifrice, in collaborazione con gli Istituti di Igiene delle Università di Modena e di Perugia; venne organizzato a Pavia, presso la Clinica Odontoiatrica, il primo Simposio del Fluoro (Zampetti e Scribante, 2020).

L'evento però a nostro giudizio più importante nell'ambito delle attività della Clinica Odontoiatrica Pavese in quegli anni fu l'interesse per l'Implantologia (Zampetti, 2003, 2006; Zampetti e Mapelli, 2004). 
Nel 1955, in collaborazione con Andrea Borghesio, assistente volontario presso la Clinica Odontoiatrica, il Palazzi, attento osservatore della nascente branca, decise di organizzare a Pavia il Primo Simposio degli Impianti Alloplastici. Vennero presentati i risultati delle ricerche della Clinica e di altri ricercatori sugli impianti alloplastici e sottoperiostei; furono qui gettate le basi della moderna implantologia con lo studio e le ricerche sperimentali su tali metodiche chirurgiche, ottenendo anche buoni risultati pratici.

E' quindi opportuno ricordare che la Scuola di Pavia fu la prima in Italia ad avviare studi sperimentali, su modello animale, sulle reazioni tissutali che gli impianti potevano determinare; fu tra le prime a considerare le esatte tecniche chirurgiche e la forma appropriata delle sottostrutture ed i risultati portati al congresso confermarono che l'implantologia doveva essere considerata una disciplina odontostomatologica, ancora certamente in evoluzione, ma che poteva essere risolutiva in problematiche complesse, a patto ovviamente di procedere con serietà nello studio della biologia, della fisiopatologia, della biocompatibilità dei tessuti e del materiale. Tali ricerche perdurano ancora al giorno d'oggi (Rizzo et al, 2009; Rizzo et al, 20ıо; Rizzo et al, 2010).

L'interesse che il Congresso (il primo nel suo genere) suscitò in Italia ed anche all'estero fu tale che pervennero al Palazzi molte richieste da parte di professionisti che volevano approfondire questa tematica.

Vennero altresì organizzati Corsi di Perfezionamento in Implantologia (tenuti da Borghesio, Branchini e De Rysky) presso la Clinica Odontoiatrica Pavese che fu anche sede di una Associazione Internazionale Europea sugli impianti

In questi anni è inoltre da ricordare l'efficace propaganda e l'attiva campagna, operate da Palazzi, per il miglioramento dell'insegnamento professionale e della legislazione odontoiatrica. In questo campo fu uno strenuo sostenitore di una preparazione odontoiatrica post-laurea obbligatoria, che doveva sfociare, secondo lui, nell'istituzione di un corso di laurea in Odontoiatria o per lo meno in un obbligo di specializzazione per il medico che voleva praticare l'odontoiatria; fu avversario dell'allora imperante "principio stomatologico" che riteneva sufficiente la sola laurea in medicina per esercitare la professione odontoiatrica (Palazzi, 1962; Corradini e Zampetti, 2010).

Nei primi anni Cinquanta il Palazzi fu anche direttore dell'Istituto Stomatologico Italiano di Milano, dove istituì una didattica ragionata teorico-pratica, sul modello di quanto avveniva a Pavia (Buonassisi, ı958; Palazzi, 1948; Trovato, I952; Zampetti, 2008, 2018). 
Non è da trascurare, inoltre, fra gli anni Cinquanta e Sessanta, l'introduzione dell'ipnosi in Odontoiatria (grazie all'odontoiatra italo-inglese Pietro Pavesi). Il Palazzi fu particolarmente interessato all'applicazione di tale metodica, tanto da praticare, in ipnoanestesia, interventi quali avulsioni dentarie e asportazioni della polpa; venne anche fondato, presso la Clinica, il "Centro per gli Studi sulla Stomatologia Psicosomatica” (Zampetti e Mapelli, 2004; Zampetti, 2006, 20Ira, 20mb).

Ebbe numerosi allievi, che condusse quasi tutti alla libera docenza. Fra questi ricordiamo: Rosolino Babini, Guido Bracchetti, Nicolò Amodeo, Giuseppe Bugliari, Cinzio Branchini, Carlo Zerosi, Giovanni Hruska, Arturo Hruska, Enzo Caudana, Aldo Negrisoli, Andrea Borghesio, Antonio Baratieri, Salvatore De Rysky, Giovanni Amici, Claudio Continolo, Ernesto Piazzini, Curzio Hruska, Carlo Lasagna, Adriano Bertolini. Fra questi predilesse tre allievi in modo particolare: Cinzio Branchini, suo primo collaboratore ed aiuto, destinato a succedergli nella Direzione della Clinica, Carlo Zerosi, del quale apprezzò la serietà scientifica e la predisposizione agli studi istologici e Antonio Baratieri, che divenne in seguito direttore della Clinica Odontoiatrica dell'Università Cattolica di Roma.

Dopo un magistero durato trentasette anni, nel 1962, Silvio Palazzi lasciò la direzione della clinica; continuò tuttavia una vivace attività culturale fin quasi alla fine dei suoi giorni, polemizzando spesso, per mezzo di riviste del settore, riguardo alla legislazione odontoiatrica ed all'imminente istituzione del Corso di Laurea in Odontoiatria, da lui sostenuto già a partire dagli anni Trenta; per circa un trentennio infatti affermò la necessità di una preparazione specifica per il futuro odontoiatra, avulsa dal percorso formativo voluto dal Decreto Boselli, che riteneva bastevole la sola Laurea in Medicina e Chirurgia per l'esercizio professionale odontostomatologico; ritenendo troppo lungo il normale iter (sei anni di corso di laurea più tre di specializzazione in Odontoiatria, fondamentale ancorchè non obbligatoria), il Palazzi propose dapprima un'ipotesi formativa che comprendesse un triennio in comune con la Facoltà Medica, per poi ottenere una laurea differenziata, di natura triennale in Odontoiatria; successivamente, su modello di quanto avveniva negli Stati Uniti ed in molte Nazioni Europee, fu convinto assertore di un Corso di Laurea apposito.

Com'è noto ciò troverà piena realizzazione nel 1980 (Zampetti, 2009).

Silvio Palazzi offrì anche un contributo notevole in campo editoriale: lamentando l'assenza, sin dai primi anni Venti, di trattati o manuali di odon- 
toiatria di autori italiani, pensò di proporne uno lui stesso, che ebbe varie rielaborazioni e che costituì per anni il testo più usato sia dagli studenti, sia dai medici pratici (Figure 3 e 4).

E' da rimarcare anche il suo apporto alla divulgazione scientifica nell'ambito enciclopedico: collaborò infatti con l'Enciclopedia Italiana di Giovanni Treccani con la stesura delle voci "Carie" (IX, p. I8, I appendice, 1948) e "Odontoiatria" (XXV, p. I8I, II appendice, 1949).



Fig 3. Frontespizio del Volume I del trattato di odontologia di Silvio Palazzi e collaboratori (edizione 1964). 


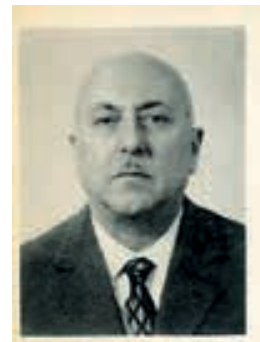

1. socisti I.echisi

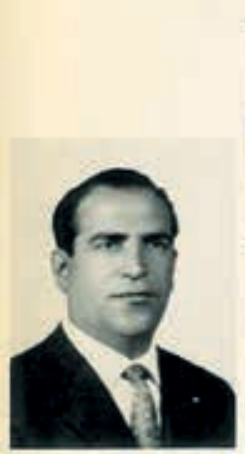

L COLA

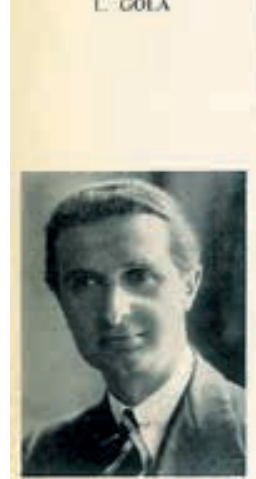

tc senemsicu
* Mat.var g. Colis.abonstori

TRATTATO

DI

ODONTOLOGIA

in Lazaose.

CON AGGIOKХAMENT AL 196\%

romes a

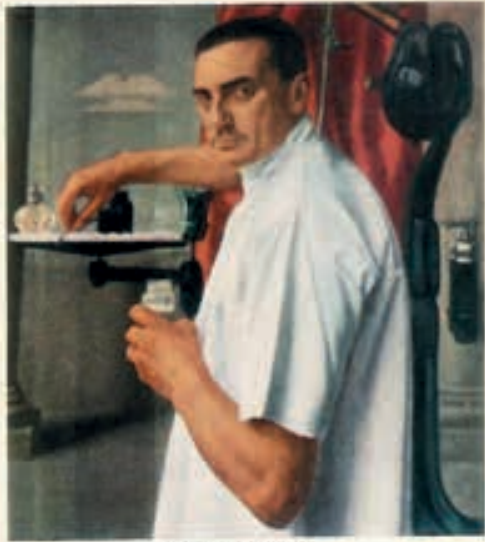

sucvo pewn
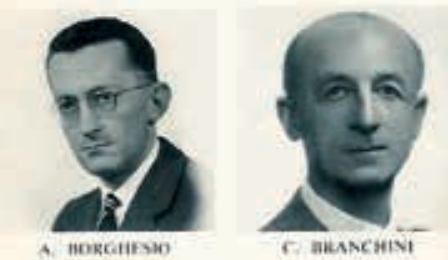

FIIZIONF HOFPI , CFNIKO STUDH IS. ,

1967

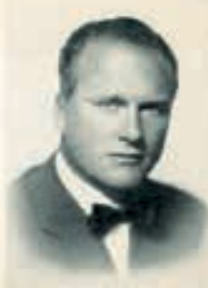

A Hetsan pariat

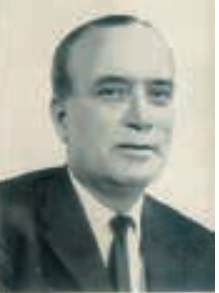

P. ravise
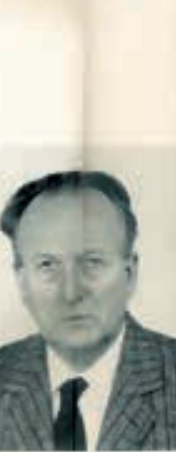

(1) ARмеснітा

Fig 4. Frontespizio del Volume I del trattato di odontologia di Silvio Palazzi e collaboratori (edizione 1964).

Forniamo di seguito l'elenco completo dei testi di Silvio Palazzi:

- Manuale di Odontologia (rg26) - prima edizione- ed. Vallardi Milano.

- Istituzioni di odontoiatria (1927) in coll. con L. Magnaghi- ed. Società editrice Libraria, Milano. 
- Trattato di Odontologia per Medici e Studenti - (1932) seconda edizione in due volumi- ed. Vallardi, Milano.

- Elementi di Odontologia (1936) in coll. con G. Fasoli Società Editrice Libraria Milano.

- Trattato Italiano di Odontologia (1942) terza edizione in tre volumi- ed Vallardi, Milano.

- Elementi di Odontologia (1944) seconda edizione in coll. con G. Fasolied. Società Editrice Libraria, Milano.

- Manuale di terapia dentaria, ortodonzia, odontotecnica (1947)- ed. Casa Editrice Ambrosiana, Milano.

- Trattato di Odontologia (1950) quarta edizione, in due volumi- ed. Hoepli, Milano.

- Trattato italiano di odontostomatologia, (1959) quinta edizione in due volumi- ed. Cortina, Milano.

- Trattato di Odontologia (rg60) in due volumi più tre di aggiornamento al r967 Hoepli, Milano..

- Prontuario di odontostomatologia in coll. con G. Briasco,(rg60)- ed. Cortina, Milano.

- Le Paradenziopatie - Palazzi e collaboratori.- (rg6I) - ed. Minerva Medica, Torino.

Sono poi da ricordare i volumi scritti dagli allievi della Scuola di Pavia:

- Carlo Zerosi - Terapia Conservativa in Odontostomatologia I942-ed. Hoepli, Milano (prima edizione); 1955- ed. Minerva Medica. Torino (seconda edizione).

- Cinzio Branchini et al.- Le malattie della bocca, dell'esofago e del duodeno, 1954-ed. Vallardi, Milano.

- Aga Hruska - La protesi multipla collegata ed incorporata, I954- ed. Sperling e Kupfer, Milano.

- Claudio Continolo - Le fratture del Condilo Mandibolare - Cortina, I955

- Angelo Sargenti - Terapia N2 - trattamento canalare razionale, i956- ed. Cortina, Milano.

- Pietro Pavesi - L'ipnosi in Odontoiatria. 1959 - ed. Cortina, Milano. 
- Carlo Zerosi - Tecnica delle preparazioni dentarie in conservativa ed in protesi; I959-ed. Cortina, Milano.

Oltre ai volumi sopra citati devono essere ricordati:

- Gli Atti del Simposio del Fluoro (1953)

- Gli scritti scientifici relativi al trentesimo anniversario di insegnamento di Silvio Palazzi (1955)

- Gli Atti del I Simposio sugli Impianti Alloplastici (1955) a cura di A. Borghesio

- Gli Atti dei Convegni sulla Terapia radicolare (1957) a cura di A. Baratieri

- Gli Atti del II Simposio sugli Impianti Alloplastici (r958) a cura di A. Borghesio

- Gli Atti del Convegno Stomatologico della Valle del Piave (1959)

- Gli Atti del I Congresso Nazionale SIOCMF (I959) a cura di A. Baratieri

$\mathrm{Ci}$ è sembrato opportuno citare in questa forma queste opere, forse anche in modo asettico e schematico ma con una visione globale, proprio per enfatizzare e rimarcare l'evidenza dell'intensa attività di tipo trattatistico-divulgativo che il Palazzi e la sua scuola operarono nel corso di un quarantennio.

\section{Conclusioni}

Si può senza dubbio affermare che Palazzi, durante dieci lustri, partecipò in prima linea a traghettare l'Odontoiatria italiana dall'era pionieristica a quella scientificamente impostata come la conosciamo al giorno d'oggi: certamente non senza polemiche o lotte personali, ma indubbiamente con passione, dedizione ed onestà intellettuale; intrattenne inoltre cordiali rapporti con le principali Cliniche Odontoiatriche europee con vivaci scambi culturali.

Alla sua morte, avvenuta nel 1979, numerosi furono i necrologi che ne ricordarono la vita e le opere: fra questi merita un cenno particolare quello scritto da Carlo Zerosi, dove traspare il commosso affetto dell'allievo verso il Maestro (Zerosi, 1979).

Certamente Silvio Palazzi è da considerarsi un gigante nella Storia dell'Odontoiatria italiana sia per quello che riuscì a realizzare in tempi complessi che per l'influsso notevole che ebbe nei confronti del modo accademico e professionale dell'epoca (Zampetti, 2009). 


\section{Bibliografia}

1. Beretta, A. (1935). Il Principio Stomatologico. Milano: Acta Medica Italica.

2. Branchini, C. (1940). Contributo della Clinica Odontoiatrica dell'Università di Pavia al progresso della disciplina in Italia. Pavia.

3. Branchini, C. (1933). L'Istituto di Odontoiatria dell'Università di Pavia. Pavia.

4. Corradini, M. \& Zampetti, P. (2010). Dentista Italiano: Storia della professione. Trento: Edizioni U.C.T.

5. Fondo Silvio Palazzi. Società Geografica Italiana. http://www.archiviofotografico.societageografica.it/index.php?it/199/fondo-silvio-palazzi.

6. Istituto Stomatologico Italiano 1908-1958 (1958). Numero unico redatto a cura di V. Buonassisi in commemorazione del cinquantenario di fondazione. Milano.

7. Palazzi, S. (1962). L'apporto della Clinica Odontoiatrica dell'Università degli Studi di Pavia ai problemi della Scuola e della legislazione per l'odontoiatria didattica e professionale. Pavia.

8. Palazzi, S. (1939). Attività clinica, scientifica, didattica, militare e politica. Pavia.

9. Palazzi, S. (1948). I quarant'anni di vita dell'Istituto Stomatologico. Rass. Trim. Odont., 3.

10. Palazzi, S. (1962). Paradenziopatie. Dottrina e pratica. Torino.

11. Rizzo, S., Zampetti, P. \& Lupi, S. M. (2010). Immediate implant periapical lesion: case report and definition of a new pathological form. Doctor Os. Apr., 21 (4), 409-415.

12. Rizzo, S., Lupi, S. M. \& Zampetti P. (2009). Nerve damage resulting from oral surgery and medical legal implications. Journal of Osseointegration, 1(3), 129-137.

13. Rizzo, S., Zampetti, P., Rodriguez, Y. Baena, R., Svanosio, D. \& Lupi S. M. (2010). Retrospective analysis of 521 endosseous implants placed under antibiotic prophylaxis and review of literature. Minerva Stomatol. Mar., 59(3), 75-88.

14. Trovato, S. (1952). Storia ed organizzazione della Scuola di Perfezionamento in Odontoiatria di Mlano, Rass. Trim. Odont., 3, 336-346.

15. Zampetti, P. \& Mapelli, P. (2004). Priorità Scientifiche dell'Istituto di Odontoiatria dell'Università di Pavia dalla fondazione agli anni Settanta: revisione storica. Bollettino della Società Medico Chirurgica di Pavia, 117(2), 59-64.

16. Zampetti, P. (2011a). Cattedre, scuole, cliniche odontoiatriche in Italia agli inizi del '900: valutazione comparativa sulle origini. Atti del X e XI Congresso Nazionale della SISOS (Società Italiana di Storia della Odontostomatologia), 2007-2009, 35-48.

17. Zampetti, P. (2011b). Gli uomini che fecero l'odontoiatria italiana: Silvio Palazzi (1892-1979). Doctor Os, 22 (9).

18. Zampetti, P. (2018). L'Istituto Stomatologico Italiano. Roma: Edizioni Aracne.

19. Zampetti, P. (2001). La clinica odontostomatologica pavese durante le direzioni di Ludovico Coulliaux, Silvio Palazzi e Cinzio Branchini. Note ergobiografiche. Bollettino della Società Pavese di Storia Patria, 391-401.

20. Zampetti, P. (2008). La fondazione dell'Istituto Stomatologico Italiano (1908): cento anni di storia. Doctor Os, 19(4), 400.

21. Zampetti, P. (2003). La Scuola Odontoiatrica Pavese. In Società Italiana di Storia della Odontostomatologia, Atti del VI Congresso Nazionale della SISOS (pp. 11-27). Pavia.

22. Zampetti, P. (2005). Ludovico Coulliaux (1863-1929), pioniere dell'odontoiatria scientifica in Italia. Doctor Os, 16(9), 1108-09. 
23. Zampetti, P. \& Scribante, A. (2020). Historical and bibliometric notes on the use of fluoride in caries prevention. European Journal of Paediatric Dentistry, 21, 148-152.

24. Zampetti, P. (2006). Silvio Palazzi (1892-1979) pioniere della moderna odontostomatologia. Doctor Os, 17(4).

25. Zampetti, P. (2009). Storia dell'Odontoiatria. Roma: Edizioni Aracne.

26. Zampetti, P. (2009). Ulteriori sviluppi dell'odontoiatria italiana nel secondo dopoguerra. Doctor Os, 20(7), 894-95.

27. Zerosi, C. (1979). In memory of Prof. Silvio Palazzi. Bull Group Int Rech Sci Stomatol Odontol., 22(4), 116-7.

\section{SuMMARY}

It is not easy to analyse a complex figure like Silvio Palazzi (I892-1979). Without a doubt, he was one of the most prominent figures in the Italian odontostomatology scene for about fifty years and one of the absolute protagonists of the transition of Italian dentistry from the pioneering era to the scientific. He was certainly a precursor and a man with an open mind, endowed with a broad vision. Palazzi had an eclectic, versatile personality, from certain points of view even brilliant but also unpredictable and difficult to understand. He was at the centre of Italian dentistry's academic and professional life; few can boast of a didactic, clinical, scientific activity like his. Having become, at a young age, the director of a clinic that was still little more than a dental practice, he was able to make it grow, revitalise it, bring it to a level of excellence that had no comparison in Italy but that could be compared to that of the great European dental clinics. He was the author of a "Treaty of Odontology" (which had seven editions) on which entire generations of dentists were formed, and he wrote over five hundred scientific publications in all the fields of Odontostomatology. He particularly favoured histological and histochemical investigations, as he often recalled, for having been trained in this sense by his attendance at the Institute of General Pathology of Pavia directed by Camillo Golgi (1843-1926, Nobel Prize for Medicine in 1906). In the clinical field, every sector of the dental discipline saw him as an attentive and passionate scholar, in particular of Endodontics and Periodontology. Furthermore, he was a pioneer of implantology when this branch received more criticism than success and began research on the prophylactic action of fluoride when many were against it. He fought assiduously for a different Italian dental legislation: he was a convinced supporter of a special Degree Course for the preparation of the future dentists, already in the Fifties. Since this project seemed difficult to carry out, he proposed, if nothing else, the requirement of a post-graduate specialisation to guarantee suitable training to dental practitioners. Despite this, due to his often aggressive and argumentative attitude, he lost the friendship of many colleagues and created numerous enemies. Certainly, he was a character who cannot go unnoticed and who, forty years after his death, deserves a careful historical evaluation.

Keywords: Palazzi, history of dentistry, University of Pavia 